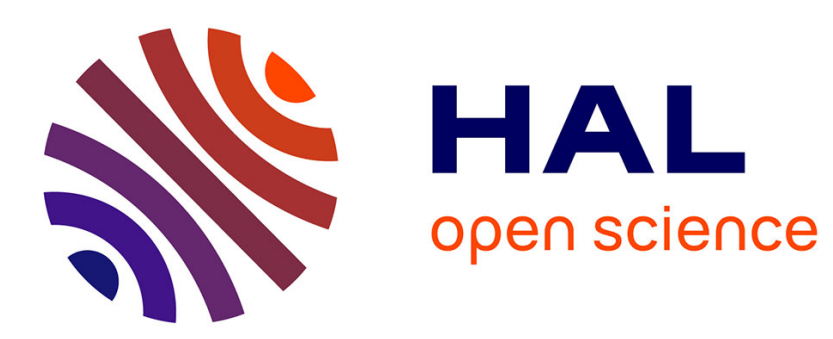

\title{
Arnaud Alessandrin, Karine Espineira (2015), Sociologie de la transphobie, Maison des sciences de l'homme d'Aquitaine. Jaine Cussac
}

\section{- To cite this version:}

Jaine Cussac. Arnaud Alessandrin, Karine Espineira (2015), Sociologie de la transphobie, Maison des sciences de l'homme d'Aquitaine.. 2017. hal-01637832

\section{HAL Id: hal-01637832 \\ https://hal.science/hal-01637832}

Submitted on 18 Nov 2017

HAL is a multi-disciplinary open access archive for the deposit and dissemination of scientific research documents, whether they are published or not. The documents may come from teaching and research institutions in France or abroad, or from public or private research centers.
L'archive ouverte pluridisciplinaire HAL, est destinée au dépôt et à la diffusion de documents scientifiques de niveau recherche, publiés ou non, émanant des établissements d'enseignement et de recherche français ou étrangers, des laboratoires publics ou privés. 


\section{Arnaud Alessandrin, Karine Espineira (2015), Sociologie de la transphobie, Maison des sciences de l'homme d'Aquitaine.}

Sociologie de la transphobie étudie le rejet et les oppressions systémiques subies par les personnes trans par opposition aux tentatives psychanalytiques objectivantes qui théorisent déjà largement des processus de transition subjectifs.

Il s'agit «d'interroger, au-delà de la question trans, les normes et les polices de genre qui s'organisent autour d'une imposition cisgenre » (p. 44). Il est en effet rappelé (p. 76) qu'une sociologie des transidentités ne peut prétendre à l'élaboration d'une définition close incluant les identifications individuelles et les spécificités des discours sociaux et culturels, à moins de se cantonner à la stricte définition psychiatrique du transsexualisme, concept médical dont l'histoire est rapportée dans le deuxième chapitre (p. 77).

Yves Raibaud souligne à ce titre, dès la préface, l'importance de la prise de parole par les personnes concernées pour produire des connaissances visant à participer aux luttes contre les discriminations faites à leur encontre.

Dévoilant les résultats statistiques de l'étude, Arnaud Alessandrin et Karine Espineira présentent différentes expériences sociales qui rendent compte de la dimension politique des oppressions transphobes (p. 35). Il est en effet nécessaire, dans une recherche de poussée égalitaire en droit, de faire la preuve de la transphobie en travaillant ses contours d'un point de vue sociologique et non uniquement juridique. Pour ce faire, les deux sociologues s'emploient à réunir également des témoignages et à réaliser une enquête quantitative dont les résultats figurent aux chapitres deux et cinq du livre.

Il est d'abord précisé que, pour concevoir les transidentités, les auteur·ice-s choisissent d'utiliser le concept de «carrière », au sens de Becker (1985), qui renvoie à «un processus d'apprentissage auquel participe pleinement le stigmate », et ce pour éviter l'écueil du processus d'essentialisation. Ainsi les récits de différentes expériences transidentitaires sontils rapportés en discernant notamment les récits «d'expérience totale », dans lesquels l'expérience transphobe semble être partout, ceux des «intouchables de la discrimination", qui tentent des mises à distance de la transphobie, et ceux des «bricoleurs », ce dernier faisant par exemple référence à l'imprévisibilité de la discrimination. Sont également distinguées les transphobies directe et indirecte, respectivement rejet explicite des transidentités et conséquence insidieuse du cisgenrocentrisme, et la transphobie institutionnelle dans le contexte médical et juridique français.

Enfin, les résultats de l'enquête quantitative menée entre mai et juin 2014 sont présentés sous forme de diagrammes qui illustrent les 309 réponses rentrées par le biais de réseaux sociaux et sites et réseaux associatifs (p. 133). Ainsi l'identité de genre, l'âge, la profession et l'orientation sexuelle des répondant.e.s sont présentés avant de distinguer les différentes formes d'oppressions subies. L'enquête révèle notamment les dénis de justice et la mauvaise gestion des plaintes par la police, ainsi que la négligence des administrations publiques et du corps médical. 
La pathologisation des personnes trans par la médecine est présentée comme une cause et une conséquence de la transphobie, dans laquelle le discours du «biopouvoir» recourt à l'assignation des personnes trans à la dysphorie de genre. Il s'agit selon les auteur.e.s de définir une prétendue maladie nécessaire à "l'attribution chichement attribuée de soins adaptés, au prix d'un humiliant parcours psychothérapeutique» (p. 13). La sphère médiatique, autre angle d'analyse du livre, véhicule quant à elle des clichés désinformants contre lesquelles les chartes pour le respect des trans luttent en indiquant notamment aux journalistes le vocabulaire approprié à adopter, et nie les crimes transphobes, que la loi française ne reconnaît pas, rendant notamment impossible toute prévention (p. 99).

À l'objection à l'étude de la transphobie qui catégoriserait les personnes discriminées ayant développé des stratégies de «vie vivable » à leurs dépens, est rappelée la nécessité de réaction des militant.e.s trans face à l'impunité de l'État devant des agressions criminelles et à «la manifestation violente, permanente et généralisée de la transphobie » (p. 14). Notons de ce point de vue que, depuis la publication du livre, la loi française a évolué en ce domaine et protège aujourd'hui les victimes de transphobie.

Le quatrième chapitre rappelle ainsi les différents mouvements militants trans depuis les années 1970 qui, en France, connaissent leur apogée en 2005 avec la deuxième édition de la marche Existrans (p. 115). Dès lors, les modes d'action se professionnalisent en multipliant les procédures à l'encontre des institutions qui refusent le changement d'état civil dont découlent l'accès au travail, à la santé, au logement ainsi qu'au mariage et ses droits familiaux à des personnes trans, mais aussi en développant l'administration des structures associatives et en produisant des connaissances expertes ou contre-expertes.

Le dernier chapitre se focalise sur l'invisibilisation des personnes trans dans les politiques de santé. Prévention et sensibilisation aux risques de MST et du VIH dans les rapports sexuels sont donc prises en charge par des militant.e.s trans comme Outrans et son fascicule Dicklit et $T$ claques et Chrysalide et son site "Gare à tes fesses » (p. 157). Il se poursuit avec une ouverture sur le travail du sexe et les personnes trans migrantes, puis quelques pistes de recherche sont suggérées comme celles du «vieillir trans », des différences entre hommes et femmes trans et entre catégories sociales et professionnelles, ainsi qu'un travail approfondi sur «les personnes trans racialisées et/ou migrantes» (p. 172).

Dans la conclusion, sans pour autant remettre en question l'importance de l'interconnexion des luttes trans avec les luttes féministes et/ou LGBI, les auteur.ice.s rappellent que la transphobie "demeure impensée », ce qui se traduit notamment par certains discours qui réassignent aux femmes trans une identité de genre masculine en prétextant qu'elles auraient été « socialisées comme des hommes » (p. 170) pour contester leur légitimité à se revendiquer d'aucun féminisme.

Finalement, l'investissement du domaine juridique, les pratiques militantes subversives et la lutte contre les stéréotypes de genre sont trois fronts distincts qui, activés simultanément, semblent proposer, selon les deux sociologues, des stratégies de lutte efficaces contre la transphobie. 
